Publ. RIMS, Kyoto Univ. Ser. A Vol. 3 (1967), pp. 139-150

\title{
Quelques remarques sur le mémoire de P. Painlevé: \\ sur les équation différentielles dont l'intégrale générale est uniforme
}

\author{
Par \\ Masuo Hukuhara
}

\section{Introduction}

Dans ses recherches sur les équations différentielles ordinaires du second ordre dont l'intégrale générale est uniforme (Bull. Soc. Math. France, 28, 1900), il a réussi, à surmonter la difficulté qui semblait insurmontable, comme l'insistait E. Picard. Pour parvenir aux résultats, il a constitué une double méthode qui permet de former des conditions nécessaires pour qu'une équation ait ses points critiques fixes et de décider si ces conditions sont ou non suffisantes.

La recherche des conditions nécessaires s'appuie sur un lemme qui affirme l'uniformité des coefficients d'un développement dont la somme est uniforme. En fait $P$. Painlevé l'a appliqué légitimement et ingénieusement. Néanmoins on doit être prudent lorsqu'on traite des fonctions multiformes admettant des lignes singulières. Pour le comprendre nous donnerons dans la première partie un contre-exemple de ce lemme.

Pour montrer que les conditions nécessaires trouvées par l'application du lemme sont suffisantes, il a formé d'abord une solution formelle qui admet un pôle mobile (formel) et dépend d'un autre paramètre et puis il démontre la convergence de la série formelle. L'équation traitée:

$$
y^{\prime \prime}=6 y^{2}+x
$$

est la plus simple de celles qui sont dépourvues de points critiques 
mobiles. Dans la deuxième partie, nous montrerons qu'il en est de même de l'équation générale du second ordre dont le second membre est une fraction rationelle en $x, y, y^{\prime}$.

\section{Sur la fonction limite des fonctions multiformes}

\section{Le lemme qui joue un rôle important.}

Il s'énonce:

Supposons que les seconds membres $H$ et $K$ du système

$$
\frac{d y}{d x}=H(x, y, z), \quad \frac{d z}{d x}=K(x, y, z),
$$

dépendent analytiquement d'un paramètre $t$ et soient holomorphes pour $t=0$; si l'intégrale générale du système (S) est uniforme quel que soit $t$ (sauf peut-être pour $t=0$ ), elle est uniforme encore pour $t=0$ et les développements de $y(x), z(x)$ suivant les puissances de $t$ ont comme coefficients des fonctions uniformes de $x$.

Ce lemme est bien intuitif et il n'y aurait aucun doute qu'il soit vrai. Mais il y aurait lieu de préciser sa signification.

D'après le théorème classique de $\mathrm{H}$. Poincaré, la solution $y(x)$, $z(x)$ du système (S) se développe suivant les puissances de $\mathrm{t}$ :

$$
\begin{aligned}
& y(x)=y_{0}(x)+t y_{1}(x)+t^{2} y_{2}(x)+\cdots \\
& z(x)=z_{0}(x)+t z_{1}(x)+t^{2} z_{2}(x)+\cdots
\end{aligned}
$$

et si $y(x)$ et $z(x)$ ainsi que $y_{0}(x)$ et $z_{0}(x)$ sont holomorphes en chaque point d'une courbe fermée $\Gamma$ (et si l'on suppose de plus que $H$ et $K$ soient holomorphes en chaque point $(x, y, z, 0)$ tel que $x \in \Gamma$, $\left.y=y_{0}(x), z=z_{0}(x)\right)$, les $y_{n}(x)$ et les $z_{n}(x)$ sont holomorphes en chaque point de $\Gamma$. Si, après avoir parcouru le chemin $\Gamma$, on trouve pour une des $y_{n}(x)$ une valeur différente de la valeur initiale, la fonction $y(x)$ ne saurait reprendre non plus sa valeur initiale pourvu que $t$ soit assez petite.

Cette démonstration du lemme suppose que $H$ et $K$ sont holomorphes en chaque point $\left(x, y_{0}(x), z_{0}(x), 0\right)$ tel que $y_{0}(x)$ et $z_{0}(x)$ soient holomorphes. Il faut donc, pour appliquer le lemme, tenir 
compte de ce fait. Car, il y aurait lieu de se proposer si $y(x)$ et $z(x)$ sont uniformes sans que les $y_{n}(x)$ et les $z_{n}(x)$ le soient. Si les $y_{n}(x)$ admettent des points critiques qui se trouvent sur une ligne $L$, ne peut-il pas arriver que la somme $y(x)$ admet $L$ comme ligne singulière et devient uniforme dans son domaine d'existence?

Nous montrerons que cette circonstance se réalise si l'on ne se restreint pas aux solutions des systèmes differentiels.

\section{Contre-exemple.}

Considérons la série de puissances en $\mathrm{t}$ :

$$
F_{t}(x)=t f_{1}(x)+t^{2} f_{2}(x)+\cdots
$$

Nous prenons pour $f_{n}(x)$ la fonction

$$
f_{n}(x)=c_{n} \sqrt{x-a_{n}},
$$

où $\left\{a_{n}\right\}$ est une suite de nombres rationnels: $1 ; 1 / 2,2 / 2,3 / 2,2 ; 1 / 3$, $2 / 3,3 / 3,4 / 3,5 / 3,6 / 3,7 / 3,8 / 3,3 ; 1 / 4, \cdots, 4 ; 1 / 5, \cdots, 5 ; \cdots . \quad \mathrm{Si}$ l'on pose

$$
\begin{aligned}
n_{k} & =1^{2}+2^{2}+\cdots+k^{2} \\
& =\frac{k}{6}(k+1)(2 k+1),
\end{aligned}
$$

on aura

$$
a_{n_{k}+m}=\frac{m}{k+1}, \quad m=1,2, \cdots,(k+1)^{2} .
$$

Nous prenons pour $\left\{c_{n}\right\}$ une suite de nombres positifs qui s'évanouit assez rapidement. Nous dirons alors que $F_{t}(x)$ est, quelle que soit la valeur $t$ différente de 0 , une fonction analytique uniforme pour laquelle le demi-axe réel positif est une ligne singulière.

En effet, soit $D$ le plan de nombres complexes coupé suivant le demi-axe réel positif $L$. Puisqu'on a

et

$$
n_{k}=O\left(k^{3}\right), a_{n}=O(k)=O\left(n^{1 / 3}\right),
$$

$$
\sqrt{x-a_{n}}=O\left(n^{1 / 6}\right),
$$

le second membre de (1) est une série qui converge uniformément à 
l'intérieur de $D$, si la série $\Sigma c_{n} n^{1 / 6}|t|^{n}$ est convergente. $F_{t}(x)$ est une fonction holomorphe dans $D$ et sa dérivée $F_{t}^{\prime}(x)$ est donnée par

$$
F_{t}^{\prime}(x)=\sum_{n=1}^{\infty} t^{n} f_{n}^{\prime}(x)
$$

Pour montrer que $L$ est une ligne singulière, il suffit de montrer que l'on a $F_{t}^{\prime}(x) \rightarrow \infty$ pour $x=i s+p / q s \rightarrow+0$, où $p / q$ est une fraction irréductible telle que $p<q^{2}$, car l'ensemble de ces fractions est dense sur $L$.

Soit $\nu$ le plus petit des indices $n$ tels que $a_{n}=p / q$. La somme

$$
\sum_{n=1}^{\nu-1} t^{n} f_{n}^{\prime}(x)
$$

est une fonction de $x$ holomorphe en $p / q$. On a d'autre part

$$
\left|1 / \sqrt{x-a_{n}}\right| \leqq\left|1 / \sqrt{x-a_{\nu}}\right|
$$

pour tous les $n$ si $x=i s+p / q$. Par suite

$$
2\left|\sum_{n=\nu+1}^{\infty} t^{n} f_{n}^{\prime}(x)\right| \leqq \sum_{n=\nu+1}^{\infty} c_{n}|t|^{n} /\left|\sqrt{x-a_{\nu}}\right| .
$$

Supposons donc, quelque grand que soit $R>0$, que l'on a

$$
\sigma c_{\nu} \geqq \sum_{k=1}^{\infty} c_{\nu+k} R^{k}
$$

pour $\nu$ assez grand, où $\sigma$ désigne un nombre positif moindre que 1 . Si l'on prend par exemple $c_{n}=1 / n !$, on a

$$
\sum_{k=1}^{\infty} c_{\nu+k} R^{k}=\frac{R}{(\nu+1) !} \sum_{k=1}^{\infty} \frac{R^{k-1}}{(\nu+2)(\nu+3) \cdots(\nu+k)}<\frac{R e^{R}}{(\nu+1) !}
$$

et la condition ci-dessus est remplie si $\sigma(\nu+1) \geqq R e^{R}$.

Si alors $|t| \leqq R$, on a

$$
2\left|\sum_{n=\nu}^{\infty} t^{n} f_{n}^{\prime}(x)\right| \geqq(1-\sigma) c_{\nu}|t|^{\nu} /|\sqrt{x-p / q}|,
$$

de sorte que

$$
\sum_{n=\nu}^{\infty} t^{n} f_{n}^{\prime}(x) \rightarrow \infty
$$

pour $x=i s+p / q, s \rightarrow+0$. C.Q.F.D. 


\section{Sur la convergence d'une série formelle qui satisfait à une équation différentielle ordinaire du second ordre}

\section{Traitement de l'équation (F) d'après $P$. Painlevé.}

Pour démontrer que la solution générale de l'équation différentielle

(F) est méromorphe, P. Painlevé utilise la transformation

$$
y=\frac{1}{z^{2}}, \quad y^{\prime}=-\frac{2}{z^{3}}-\frac{x z}{2}-\frac{z^{2}}{2}+u z^{3} .
$$

On a pour $z$ et $u$ les équations différentielles

$$
\left\{\begin{array}{l}
z^{\prime}=1+\frac{x z^{4}}{4}+\frac{z^{5}}{4}-\frac{u z^{6}}{2} \\
u^{\prime}=\frac{x^{2} z}{8}+\frac{3 x z^{2}}{8}+\frac{z^{3}}{4}-\left(x z^{3}+\frac{5 z^{4}}{4}\right) u+\frac{3}{2} u^{2} z^{5} .
\end{array}\right.
$$

Ce système différentiel admet une solution holomorphe telle que $z(a)$ $=0, u(a)=h$, $a$ et $h$ désignant des valeurs arbitraires. En portant cette solution dans la transformation $(T)$, on obtient la solution qui admet un pôle moile $a$ et dépend d'une autre constante arbitraire $h$. La transformation est obtenue comme il suit.

On détermine d'abord une série

$$
y=\frac{c}{(x-a)^{2}}+\frac{c^{\prime}}{x-a}+c_{1}(x-a)+c_{2}(x-a)^{2}+\cdots,
$$

qui satisfait formellemant à l'équation (F) et on trouve

$$
\begin{aligned}
& c=1, \quad c^{\prime}=c_{0}=c_{1}=0, \quad c_{2}=-a / 10, \quad c_{3}=-1 / 6, \\
& c_{4}=h, \quad c_{5}=0, \quad c_{6}=a^{2} / 300, \cdots .
\end{aligned}
$$

On transforme la solution formelle en une série de puissances de $x-a$ dont les coefficients sont indépendants de $a$ (mais dépendent naturellement de $x$ ). On obtient ainsi pour $y$ et $y^{\prime}$ les séries formelles

$$
\begin{aligned}
& y=\frac{1}{(x-a)^{2}}-\frac{x}{10}(x-a)^{2}-\frac{1}{15}(x-a)^{3}+h(x-a)^{4}+\cdots \\
& y^{\prime}=-\frac{2}{(x-a)^{3}}-\frac{x(x-a)}{5}-\frac{3}{10}(x-a)^{2}+4 h(x-a)^{3}+\cdots .
\end{aligned}
$$


Eliminant $x-a$ entre ces deux relations, on obtient une relation formelle

$$
y^{\prime}=-\frac{2}{\varepsilon z^{3}}-\frac{x_{\varepsilon} z}{2}-\frac{z^{2}}{2}+7 h_{\varepsilon} z^{3}+\cdots \quad(\varepsilon= \pm 1),
$$

où la variable $z$ est liée à $y$ par $y=1 / z^{2}$. La transformation est obtenue, en supprimant du second membre les termes des degrés supérieurs à 3 et en prenant $\varepsilon=1, h=u$.

On obtient ainsi pour $u$ et $z$ des équations différentielles dont les seconds membres sont des fonctions holomorphes pour $x=a, z=0, u$ $=h$. On en conclut la convergence de la solution formelle et l'existence du pôle mobile est démontrée.

\section{Explication du problème.}

Proposons-nous maintenant la question suivante.

Considérons l'équation différentielle

$$
y^{\prime \prime}=R\left(x, y, y^{\prime}\right)
$$

où

$$
R\left(x, y, y^{\prime}\right)=P\left(x, y, y^{\prime}\right) / Q\left(x, y, y^{\prime}\right)
$$

est une fraction rationnelle irréductible en $x, y, y^{\prime}$ et supposons l'existence d'une solution formelle

$$
y=(x-a)^{-m} \sum_{n=0}^{\infty} c_{n}(x-a)^{n}, \quad\left(m>0, c_{0} \neq 0\right) .
$$

Si les coefficients $c_{n}$ dépendent en outre de a d'un autre paramètre $h$, est-elle convergente pour $x-a$ assez petit, lorsque $h$ appartient à un certain domaine?

On peut écrire

$$
\begin{aligned}
& P\left(x, y, y^{\prime}\right)=\sum A_{i j l}(a)(x-a)^{i} y^{j} y^{\prime \prime}, \\
& Q\left(x, y, y^{\prime}\right)=\sum B_{i j l}(a)(x-a)^{i} y^{j} y^{\prime \prime}
\end{aligned}
$$

et pour déterminer $c_{0}$, on obtient une relation

$$
\begin{aligned}
& -\sum^{\prime}(-m)^{l+1}(m+1) B_{i j l}(a) c_{0}^{j+l+1} \\
& =\Sigma^{\prime \prime}(-m)^{l} A_{i j l}(a) c_{0}^{j+l},
\end{aligned}
$$


où la sommation $\Sigma^{\prime}$ est étendue pour toutes les combinaisons $(i, j, l)$ telles que les ordres $-i+j m+l(m+1)+m+2$ des termes correspordants sont les plus élevés et la sommation $\Sigma^{\prime \prime}$ a la signification analogue, les ordres correspondants des deux membres étant égaux.

Deux cas sont à distinguer suivant que (4.2) est une identité ou une relation algébrique en $c_{0}$.

Dans le premier cas $c_{0}=c$ est une constante arbitraire. Dans le second cas $c_{0}$ est une fonction algébrique du paramètre $a$.

D'une manière générale, pour déterminer $c_{n}$, on obtient une relation

$$
\begin{aligned}
c_{n} \sum^{\prime} & (-m)^{l}\{j m(m+1)-l(m+1)(n-m) \\
& +(n-m)(n-m-1)\} B_{i j k}(a) c_{0}^{j+l} \\
= & c_{n} \sum^{\prime \prime}(-m)^{l-1}\{-j m+l(n-m)\} A_{i j k}(a) c_{0}^{j+k-1}+\cdots,
\end{aligned}
$$

où les termes non écrits ne dépendent que de $c_{0}, c_{1}, \cdots, c_{n-1}$. Supposons que ces coefficients aient été déjà définis comme fonctions algébriques de $a$. La relation (4.3) détermine en général $c_{n}$ d'une maniére unique comme fonction de $a$. Si elle ne détermine pas $c_{n}$ d'une manière unique, on trouvera une identité ou une impossibilité. Si elle est une identité, $c_{n}$ est une constante arbitraire. Si elle est une impossibilité on ne pourra obtenir la solution formelle (4.1). Cette hypothèse doit donc être rejetée.

Supposons qu'un des coefficients $c_{0}, c_{1}, \cdots, c_{n-1}$ peut prendre une valeur quelconque $c$. En général la relation (4.3) détermine $c_{n}$ d'une manière unique comme fonction algébrique en $a$ et rationnelle en $c$. Si elle est une identité, $c_{n}=c^{\prime}$ est aussi une constante arbitraire. On peut considérer encore un cas possible: $c_{n}$ disparaît dans la relation mais la somme des termes non écrits ne s'évanouit pas. Alors on doit déterminer le paramètre $c$ de manière que la somme des termes non écrits s'évanouisse. $c$ est alors une fonction algébrique de $a$ et $c_{n}=c^{\prime}$ est un nouveau paramètre.

On pourra ainsi déterminer de proche en proche les coefficients $c_{k}$ algébriquement, et l'un d'eux, soit $c_{\nu}=h$, est un paramètre; les 
coefficients qui le précèdent sont des fonctions algébriques d'une seule variable $a$ et ceux qui lui succèdent sont des fonctions algébriques en $a$ et rationnelles en $h$.

5. Le cas où le premier coefficient $c_{0}$ est une constante arbitraire h.

Posons

$$
y=u / z^{m}, \quad y^{\prime}=-m u / z^{m+1},
$$

On aura alors pour $z$ et $u$ les équation différentielles

$$
\left\{\begin{array}{l}
m u z^{\prime}=z^{m+2} R\left(x, u / z^{m},-m u / z^{m+1}\right)-m^{2} u, \\
z u^{\prime}=z^{m+2} R\left(x, u / z^{m},-m u / z^{m+1}\right)-m(m+1) u .
\end{array}\right.
$$

En résolvant les équation (5.1) par rapport à $z$ et $u$, on obtient

$$
z=-m y / y^{\prime}, \quad u=(-m)^{m} y^{m+1} / y^{\prime m} \text {. }
$$

En portant les séries formelles

$$
y=(x-a)^{-m} \sum_{n=0}^{\infty} c_{n}(x-a)^{n}, \quad y^{\prime}=(x-a)^{-m-1} \sum_{n=0}^{\infty}(n-m) c_{n}(x-a)^{n}
$$

dans les seconds membres de (5.3), on obtient une solution formelle du système différentiel (5.2):

$$
z=\sum_{n=0}^{\infty} \alpha_{n}(x-a)^{n}, \quad u=\sum_{n=0}^{\infty} \beta_{n}(x-a)^{n},
$$

où $\beta_{0}=h, \alpha_{1}=1$ et en général $\alpha_{k}$ et $\beta_{k}$ sont des polynomes en $h, c_{1}$, $\cdots, c_{k}$ et $1 / \mathrm{h}$. Par suite, ils sont des fonctions rationnelles en $h$ et algébriques en $a$.

\section{Le cas où $c_{\nu}(\nu>0)$ est une constante arbitraire $\boldsymbol{h}$.}

La solution formelle peut se transformer en une série formelle de puissances en $x-a$ dont les coefficients ne dépendent que de $h$ et de $x$ :

$$
y=(x-a)^{-m} \sum_{n=0}^{\infty} \gamma_{n}(x-a)^{n}
$$

et on voit immédiatement que l'on a 


$$
r_{n}(x)=c_{n}(x)-c_{n-1}^{\prime}(x)+\cdots+\frac{(-1)^{n}}{n !} c_{0}^{(n)}(x),
$$

où $c_{i}^{(j)}$ désigne la dérivée $j^{\text {iéme }}$ de $c_{i}$ considéré comme fonction de $a$. On a en particulier

$$
r_{\nu}=h+\text { fonction algébrique de } x .
$$

Les $\gamma_{n}$ sont des fonctions rationnelles en $h$ et algébriques en $x$, ceux qui précèdent $\gamma_{\nu}$ étant indépendants de $h$. En différentiant la relation (6.1) par rapport à $x$, on obtient

$$
y^{\prime}=(x-a)^{-m-1} \sum_{n=0}^{\infty}\left(\gamma_{n-1}^{\prime}+(n-m) r_{n}\right)(x-a)^{n} .
$$

L'élimination de $x-a$ entre les deux relations (6.1) et (6.1)' nous amène à une relation

$$
y^{\prime}=\sum_{n=0}^{\infty} \rho_{n}(\omega z)^{n-m-1}, \quad\left(\omega^{m}=1\right),
$$

en posant $y=1 / z^{m}$.

On a en effet

$$
\omega z=(x-a)\left\{\sum_{n=0}^{\infty} r_{n}(x-a)^{n}\right\}^{-1 / m},
$$

d'où l'on obtient pour $x-a$ une série formelle de puissances en $\omega z$ :

$$
x-a=c_{0}(x)^{1 / m}(\omega z)\left[1+\cdots+\left\{\frac{r_{n}(x)}{m c_{0}(x)^{\frac{m-1}{m}}}+\cdots\right\}(\omega z)^{n}+\cdots\right] .
$$

En portant cette expression dans le second membre de la relation (6. 1)', on obtient la relation (6.2), et on voit sans peine

et

$$
\rho_{0}=-m / c_{0}(x)^{1 / m}
$$

$$
\rho_{n}=\frac{(n-m) c_{0}(x)^{\frac{n-1}{m}+m+1}}{c_{0}(x)} \cdot \gamma_{n}+\cdots, n \supseteqq 1,
$$

où les termes non écrits ne dépendent que de $\gamma_{0}, \cdots, \gamma_{n-1}$.

Effectuons maintenant la transformation

$$
y=1 / z^{m}, \quad y^{\prime}=z^{-m-1}\left\{\sum_{n=0}^{\nu-1} \rho_{n} z^{n}+u z^{\nu}\right\} .
$$

On obtient pour $z$ et $u$ des équations différentielles de forme normale dont les seconds membres sont des fonctions rationnelles en $z$, $u$ et 
algébrique en $x$. Nous disons qu'elles admettent une solution formelle (5. 4).

En effet, l'élimination entre les deux relations

$$
\left\{\begin{array}{l}
z=(x-a)\left\{\sum_{n=0}^{\infty} r_{n}(x-a)^{n}\right\}^{-1 / m} \\
y^{\prime}=(x-a)^{-m} \sum_{n=0}^{\infty}\left(\gamma_{n-1}^{\prime}+(n-m) r_{n}\right)(x-a)^{n}
\end{array}\right.
$$

nous amène à la relation

$$
y^{\prime}=\sum_{n=0}^{\infty} \rho_{n} z^{n-m-1} .
$$

Cette relation est donc satisfaite si l'on prend

$$
u=\sum_{n=\nu}^{\infty} \rho_{n} z^{n-\nu}
$$

En portant la première des expressions (6.4) dans cette relation et en développant $u$ et $z$ ainsi obtenues suivant les puissances de $x-a$, nous obtenons les expressions (5.4), les $\alpha_{n}$ et les $\beta_{n}$ sont des fonctions algébriques de $a$ et on a en particulier

$$
\alpha_{1}=\gamma_{1}(a)^{-1 / m}, \quad \beta_{0}=(\nu+1) \gamma_{\nu}(a) / c_{0}(a)^{1 / m}+\cdots .
$$

L'assertion est donc vérifiée. $\gamma_{\nu}(a)$ dépendant de $h, \beta_{0}$ aussi peut prendre des valeurs arbitraires. Les autres $\alpha_{k}$ et $\beta_{k}$ sont des fonctions rationnelles en $h$ et algébriques en $a$.

\section{Un lemme.}

Avant de démontrer la convergence des séries (5.4), établissons un lemme.

Lemme. Considérons un système de deux équations différentielles

$$
x^{\lambda} \cdot \frac{d y}{d x}=f(x, y, z), \quad x^{\lambda} \cdot \frac{d z}{d x}=g(x, y, z),
$$

où $\lambda$ est un entier et les seconds membres sont des fonctions holomorphes de $x, y, z$ dans un voisinage de $(0,0,0)$ et telles que l'une au moins des valeurs $f\left(0, h_{0}, k_{0}\right), g\left(0, h_{0}, k_{0}\right)$ est différente de 0 :

$S$ 'il admet une solution formelle 


$$
y=\sum_{n=0}^{\infty} p_{n} x^{n}, \quad z=\sum_{n=0}^{\infty} q_{n} x^{n},
$$

dont les coefficients sont des fonctions de deux constantes arbitraires $p_{0}=h, q_{0}=k$ holomorphes pour $h=h_{0}, k=k_{0}$, on a nécessairement $\lambda \leqq 0$.

Si $\lambda \leqq 0$, le système différential (7.1) admet, d'après un théorème bien classique, une solution telle que $y(0)=h ; z(0)=k$ et elle est une fonction de $x, h, k$ holomorphe dans un voisinge de $\left(0, h_{0}, k_{0}\right)$. $\operatorname{Par}$ suite les séries (7.2) convergent lorsque les valeurs $x, h-h_{0}, k-k_{0}$ sont assez petites.

La démonstration est immédiate.

En effet, si l'on porte les séries $(7 \cdot 2)$ dans les équations $(7 \cdot 1)$, les deux membres se développent formellement en des séries de puissances de $x$ et si $\lambda>0$, les premiers termes seraient 0 dans les premiers membres et $f(0, h, k) g(0, h, k)$ dans les seconds membres. On aurait donc une contradiction puisque l'une au moins des deux valeurs $f\left(0, h_{0}, k_{0}\right)$ et $g\left(0, h_{0}, k_{0}\right)$ est différente de 0 .

\section{Convergence de la solution formelle.}

Revenons au système différentiel en $z, u$ lequel nous écrivons

$$
\frac{d z}{d x}=F(x, z, u), \quad \frac{d u}{d x}=G(x, z, u) .
$$

Les seconds membres sont des fonctions rationnelles en $z, u$ et algébriques en $x$. Si l'on prend $z$ pour variable indépendante, on obtient

$$
\frac{d x}{d z}=\frac{1}{F(x, z, u)}, \quad \frac{d u}{d z}=\frac{G(x, z, u)}{F(x, z, u)} .
$$

D'autre part, puisque $\alpha_{1} \neq 0$, la première des relations (5.4) nous permet de résoudre $x$ comme série entière de $z$ et en la portant dans la deuxième des relations (5.4), on obtient pour $u$ une série entière de $z$; soient

$$
x=\sum_{n=0}^{\infty} p_{n} z^{n}, \quad u=\sum_{n=0}^{\infty} q_{n} z^{n}
$$


les séries formelles ainsi obtenues. Les coefficients sont des fonctions algébriques en $p_{0}=a$ et $q_{0}=k$. Les systèmes des valeurs $(a, k)$ où les $p_{n}$ et $q_{n}$ deviennent singuliers forment un ensemble au plus dénombrable. Si $\left(a_{0}, k_{0}\right)$ n'appartient pas à cet ensemble, le lemme établi au $\mathrm{n}^{\circ}$ précédent est applicable et les séries (8.3) sont convergentes lorsque les valeurs $z, a-a_{0}$ et $k-k_{\mathrm{c}}$ sont assez petites.

Les variables $z$ et $u$ considérées comme fonctions de $x$ sont holomorphes dans un voisinage de $a_{0}$ et on a les développements (5.4) qui convergent lorsque $a-a_{0}$ et $h-h_{0}$ sont assez petites. En les portant dans (5.1) nous trouvons une solution qui admet un pôle mobile $a$ et dépend de la constante arbitraire $h$. Le problème posé au $\mathrm{n}^{\circ} 4$ est donc résolu affirmativement. 